\title{
Performance of LCD iterative method in the finite element and finite difference solution of convection-diffusion equations
}

\author{
L. Catabriga ${ }^{1, * \dagger}$, A. M. P. Valli ${ }^{1, \ddagger}$, B. Z. Melotti ${ }^{1, \S}$, \\ L. M. Pessoa ${ }^{1, \uparrow}$ and A. L. G. A. Coutinho ${ }^{2, \|}$ \\ ${ }^{1}$ Department of Computer Science, Federal University of Espirito Santo, Brazil \\ ${ }^{2}$ Center for Parallel Computations and Department of Civil Engineering-COPPE, \\ Federal University of Rio de Janeiro, Brazil
}

\begin{abstract}
SUMMARY
In this work, we evaluate the performance of the left conjugate direction method (LCD) for the solution of non-symmetric systems of linear equations arising from finite element and finite difference discretizations of the convection-diffusion equation. We extend the LCD algorithm proposed by Dai and Yuan (Int. J. Numer. Meth. Engng 2004; 60:1383-1399) to accommodate restarts. Our discussion considers comparison studies between the computational efficiency of the GMRES and LCD methods and some issues related to the choice of the forcing term in the inexact Newton method. Copyright (C) 2005 John Wiley \& Sons, Ltd.
\end{abstract}

KEY WORDS: left conjugate direction method; iterative solvers; inexact Newton method

\section{INTRODUCTION}

Numerical strategies for flow problems in science and engineering often requires repeated solution of non-linear systems of equations involving millions of unknowns. After some form of linearization, these systems are usually solved by Krylov subspace iterative methods [1]. Yuan et al. [2] introduced a new algorithm for solving non-symmetric, non-singular linear systems, the left conjugate direction (LCD for short) method. This method is based on the concept of left and right conjugate vectors for non-symmetric and non-singular matrices and

\footnotetext{
*Correspondence to: L. Catabriga, Av. Fernando Ferrari, 514, Goiabeiras, ES 29075-910, Vitória, Brazil.

†E-mail: luciac@inf.ufes.br

‡E-mail: avalli@inf.ufes.br

§E-mail: bzmelotti@inf.ufes.br

IE-mail: lmantovaneli@hotmail.com

"E-mail: alvaro@nacad.ufrj.br

Contract/grant sponsor: $\mathrm{CNPq} / \mathrm{MCT}$

Contract/grant sponsor: Federal University of Espírito Santo

Contract/grant sponsor: CAPES-University of Texas
}

Received 9 May 2005

Copyright (c) 2005 John Wiley \& Sons, Ltd.

Revised 23 August 2005

Accepted 28 October 2005 
possesses several theoretical advantages: (i) it has a finite termination property; (ii) breakdown for general matrices can be avoided and (iii) there is a connection between LCD and LU decomposition. Initial experiments in Reference [2] using a MATLAB implementation have shown that LCD has attractive convergence rates when compared to Bi-CGSTAB, QMR and GMRES algorithms.

Catabriga et al. [3] evaluated the performance of the original LCD algorithm in the solution of non-symmetric systems of linear equations arising from the implicit semi-discrete SUPG finite element formulation for inviscid compressible flows described in Reference [4]. They extended the original algorithm to accommodate restarts and typical finite element preconditioners. Comparisons with other Krylov subspace methods with or without preconditioning unfortunately did not favour the LCD method. Although requiring usually less iterations, CPU times and memory are larger than GMRES, Bi-CGSTAB and TFQMR. The main reason is the need to compute two matrix-vector products per iteration, one with the coefficient matrix and the other with its transposed matrix.

Valentim et al. [5] studied the solution of non-linear systems using an inexact Newton method where the approximate solution of the resulting linear system at each iteration is obtained by LCD or GMRES. A spatial discretization based on centred finite difference approximations of the heat equation and the convection-diffusion equation was considered. They studied the computational efficiency of the two linear solvers and some issues related to the choice of the forcing term in the inexact Newton method. The results have shown that the LCD method is faster than the GMRES method in most of the cases.

Recently, Dai and Yuan [6] proposed a new technique to overcome the breakdown problem appearing in the semi-conjugate direction method and a memory limitation scheme similar to the limited-memory BFGS method to minimize memory requirements of the original algorithm. In this work, we introduce restarts on the new LCD algorithm given by Dai and Yuan [6] and compare it with the restarted LCD algorithm given by Catabriga et al. [3] and the restarted GMRES method for the solution of the linear and non-linear problems discretized by finite element and finite difference methods. For the non-linear problems we study the choice of the forcing term of the inexact Newton method.

The remainder of this work is organized as follows. In the next section, we briefly review the stabilized finite element formulation for linear convection equation and the finite difference discretization of the non-linear convection equation. Section 3 introduces the inexact Newton method and shows the forcing term evaluation strategies. In Section 4, we describe the LCD algorithms, with particular emphasis on the introduction of restarts. Section 5 shows several numerical experiments, where we compare the performance of the LCD method with the GMRES method. Finally the paper ends with a summary of our main conclusions.

\section{GOVERNING EQUATIONS AND DISCRETE FORMULATIONS}

\subsection{Linear convection-diffusion equation}

Let us consider the following convection-diffusion equation defined in a domain $\Omega$ with boundary $\Gamma$ :

$$
\boldsymbol{\beta} . \nabla u-\nabla \cdot(\boldsymbol{\kappa} \nabla u)=f
$$




$$
\begin{aligned}
u=g & \text { on } \Gamma_{g} \\
\text { ก.к } \nabla u=h & \text { on } \Gamma_{h}
\end{aligned}
$$

where $u$ represents the quantity being transported (e.g. temperature, concentration), $\boldsymbol{\beta}$ is the divergence-free flow velocity and $\boldsymbol{\kappa}$ is the volumetric diffusivity. Equations (2) and (3) are the essential and natural boundary conditions, respectively, $g$ and $h$ are given functions of $\mathbf{x}=(x, y)$, and $\mathbf{n}$ is the unit outward normal vector at the boundary, $\Gamma_{g}$ and $\Gamma_{h}$ are the complementary subsets of $\Gamma$ where boundary conditions are prescribed.

Consider a finite element discretization of $\Omega$ into elements $\Omega_{e}, e=1, \ldots, n_{\mathrm{el}}$, where $n_{\mathrm{el}}$ is the number of elements. The stabilized finite element formulation of Equation (1), described in detail in Reference [7], leads to a system of linear equations

$$
\mathbf{K v}=\mathbf{F}
$$

where $\mathbf{v}=\left\{u_{1}, u_{2}, \ldots, u_{n \text { nodes }}\right\}^{t}$ is the vector of nodal values of $u, \mathbf{K}$ is called the 'stiffness' matrix and $\mathbf{F}$ is the 'load' vector.

\subsection{Non-linear convection-diffusion equation}

Let us consider the non-linear convection-diffusion equation defined in a square domain $\Omega=\left(0, l_{x}\right) \times\left(0, l_{y}\right)$ with boundary $\Gamma$

$$
\begin{aligned}
\phi u \nabla \cdot u-\nabla^{2} u & =f \\
u & =g \quad \text { on } \Gamma
\end{aligned}
$$

where $u$ represents again the quantity being transported, functions $f(x, y), g(x, y)$ and constant $\phi$ are known. Consider a discretization of $\Omega$ into a uniform grid with $n+2$ points in the $x$ direction and $m+2$ points in the $y$ direction, i.e.

$$
x_{i}=i \times h_{x}, \quad i=0, \ldots, n+1 \quad y_{j}=j \times h_{y}, \quad j=0, \ldots, m+1
$$

where $h_{x}=l_{x} /(n+1)$ and $h_{y}=l_{y} /(m+1)$. Since the values at the boundaries are known, we have $N=n \times m$ unknowns points in $\Omega$. We consider the approximation of the first- and secondorder derivatives by centred finite differences, arriving to the non-linear system of equations

$$
F\left(u_{1}, u_{2}, \ldots, u_{N}\right)=\left[\begin{array}{c}
f_{1}\left(u_{1}, u_{2}, \ldots, u_{N}\right) \\
\vdots \\
f_{N}\left(u_{1}, u_{2}, \ldots, u_{N}\right)
\end{array}\right]=\left[\begin{array}{c}
0 \\
\vdots \\
0
\end{array}\right]
$$

where $F: \mathbb{R}^{N} \rightarrow \mathbb{R}^{N}$ is a non-linear vector function, $u_{1}, u_{2}, \ldots, u_{N}$ are the unknowns and each $f_{k}$ depends only the unknowns $u_{k-n}, u_{k-1}, u_{k}, u_{k+1}$ and $u_{k+n}$ for $k=1,2, \ldots, N$.

\section{THE INEXACT NEWTON METHOD}

The non-linear system (8) can be solved by Newton's method. It is an iterative method for non-linear equations that approximate the function $F$ at a given point $\mathbf{u}=\left(u_{1}, u_{3}, \ldots, u_{N}\right)^{t}$ by a 
linear function. The Jacobian matrix $J$ represents the variation of the function $F$ with respect of $\mathbf{u}$. Each iteration of the Newton's method is given by

$$
\mathbf{u}^{k+1}=\mathbf{u}^{k}+\mathbf{s}^{k}
$$

where $\mathbf{s}^{k}$ is calculated by the solution of the linear system

$$
J\left(\mathbf{u}^{k}\right) \mathbf{s}^{k}=-F\left(\mathbf{u}^{k}\right)
$$

We may terminate the iteration when the relative non-linear residual $\left\|F\left(\mathbf{u}^{k}\right)\right\| /\left\|F\left(\mathbf{u}^{0}\right)\right\|$ is small. However, if there is error in evaluation of $F$ or the initial iterate is near a solution, a termination decision based on the relative residual may be made too late in the iteration or it may not terminate at all. Kelley [8] suggested to stop the iteration if

$$
\left\|F\left(\mathbf{u}^{k}\right)\right\| \leqslant \tau_{\mathrm{r}}\left\|F\left(\mathbf{u}^{0}\right)\right\|+\tau_{\mathrm{a}}
$$

where the relative error tolerance $\tau_{\mathrm{r}}$ and absolute error tolerance $\tau_{\mathrm{a}}$ are input to the algorithm. When an iterative method is used to solve system (10), the resulting method is known as inexact Newton method [9]. Inexact Newton methods are especially well suited for large-scale problems and have been used very successfully in many applications. In this work, we use two schemes for choosing adaptively tolerances for the inner iterative method, or the forcing term, one suggested by Papadrakakis [10] and other suggested by Kelley [8]. The calculation of the linear system tolerances suggested by Papadrakakis [10] is given by

$$
\eta_{k}=\min \left\{\eta_{\max },\left(\frac{\left\|F\left(\mathbf{u}^{k}\right)\right\|}{\left\|F\left(\mathbf{u}^{0}\right)\right\|}\right)^{v}\right\}
$$

where $\eta_{\max }$ and $0<v<1$ are known parameters. In this work we consider $\eta_{\max }=0.9999$ and $v=0.5$. On the other hand, Kelley [8] suggested that the convergence was superlinear using the values

$$
\eta_{k}= \begin{cases}\eta_{\max }, & k=0 \\ \min \left(\eta_{\max }, \eta_{k}^{A}\right), & k>0 \text { and } \gamma \eta_{k-1}^{2}<0.1 \\ \min \left(\eta_{\max }, \max \left(\eta_{k}^{A}, \gamma \eta_{k-1}^{2}\right)\right), & k>0 \text { and } \gamma \eta_{k-1}^{2}>0.1\end{cases}
$$

where the $\eta_{\max }$ is an upper limit on the sequence $\eta_{k}, k=1,2, \ldots$ The constant 0.1 is somewhat arbitrary and $\eta_{k}^{A}=\gamma\left(\left\|F\left(\mathbf{u}^{k}\right)\right\|^{2} /\left\|F\left(\mathbf{u}^{k-1}\right)\right\|^{2}\right)$. The initial values suggested by Kelley [8] are $\gamma=0.9$ and $\eta_{\max }=0.9999$.

\section{THE LEFT CONJUGATE DIRECTION ALGORITHM}

The finite element discretization of the linear convection-diffusion equation and the finite difference discretization of the non-linear convection-diffusion equation described before yields a linear problem given in (4) and a non-linear problem given in (8). In any case we have to solve a system of linear equations of the form

$$
A x=b
$$


where $A$ is an $N \times N$ non-symmetric sparse matrix, $x$ is the vector of nodal unknowns and $b$ is a known vector.

The LCD method was recently introduced in Reference [2]. In this method vectors $p_{1}$, $p_{2}, \ldots, p_{N} \in \mathbb{R}^{N}$ are called left conjugate direction vectors of an $N \times N$ real non-singular matrix $A$ if

$$
\begin{array}{ll}
p_{i}^{\mathrm{T}} A p_{j}=0 & \text { for } i<j \\
p_{i}^{\mathrm{T}} A p_{j} \neq 0 & \text { for } i=j
\end{array}
$$

Suppose that the solution of system (14) is $x^{*}$, and $\left\{p_{1}, p_{2}, \ldots, p_{N}\right\}$ are left conjugate direction vectors of $A$. Then it follows that

$$
x^{*}=x_{0}+\sum_{i=1}^{N} \alpha_{i} p_{i}
$$

for every fixed vector $x_{0}$. If $r$ denotes the residual vector then

$$
r=r_{0}-\sum_{i=1}^{N} \alpha_{i} A p_{i}
$$

where $r_{0}$ is the initial residual vector. To determine $\alpha_{i}$, since $p_{1}, p_{2}, \ldots, p_{N}$ are linearly independent, then take $r$ orthogonal to all $p_{i}$, that is

$$
p_{i}^{\mathrm{T}} r=0 \quad \forall i=1, \ldots, N
$$

From (18) we obtain

$$
\alpha_{i}=\frac{p_{i}^{\mathrm{T}} r_{i-1}}{p_{i}^{\mathrm{T}} A p_{i}}
$$

We also can write

$$
\begin{aligned}
& r_{i}=b-A x_{i}=r_{i-1}-\alpha_{i} A p_{i} \\
& x_{i}=x_{0}+\sum_{k=1}^{i} \alpha_{k} p_{k}=x_{i-1}+\alpha_{i} p_{i}
\end{aligned}
$$

From (19)-(21) we can implement the left conjugate direction method if we know the set of linearly independent vectors $p_{1}, p_{2}, \ldots, p_{N}$ such that they are left conjugate direction vectors of $A$. There is still a recurrence relation among $p_{1}, p_{2}, \ldots, p_{k}$ and $r_{k}$ to compute the left conjugate gradient vector $p_{k+1}$. For this, we need to know the first vector $p_{1}$ such that $p_{1}^{\mathrm{T}} A p_{1} \neq 0$. Yuan et al. [2] described the complete left conjugate direction method as follows.

\section{Algorithm 4.1}

1. Input $x, A, p_{1}$ such that $p_{1}^{\mathrm{T}} A p_{1} \neq 0$ and $b$;

2. $r=b-A x$;

3. For $k=1, \ldots, N$ do

$$
\begin{aligned}
& \text { 3.1. } q_{k}=A^{\mathrm{T}} p_{k}, \\
& \alpha_{k}=p_{k}^{\mathrm{T}} r / q_{k}^{\mathrm{T}} p_{k}, \\
& x=x+\alpha_{k} p_{k}, \\
& r=r-\alpha_{k} A p_{k} ;
\end{aligned}
$$




$$
\text { 3.2. } \begin{aligned}
& p_{k+1}=r, \\
& \beta_{i}=-q_{i}^{\mathrm{T}} p_{k+1} / q_{i}^{\mathrm{T}} p_{i}, \\
& p_{k+1}=p_{k+1}+\beta_{i} p_{i} \text { for } i=1, \ldots, k .
\end{aligned}
$$

In Algorithm 4.1 we need to store $N$ vectors $p_{k}$ and $N$ vectors $q_{k}$, furthermore we need two matrix-vector product per iteration to obtain the solution $x$. Dai and Yuan [6] proposed new ideas for the LCD methods where one matrix-vector product is needed. The new algorithm can be written as follows.

\section{Algorithm 4.2}

1. Input $x, A, p_{1}$ such that $p_{1}^{\mathrm{T}} A p_{1} \neq 0$ and $b$;

2. $r=b-A x$;

3. $q_{1}=A p_{1}$;

4. For $k=1, \ldots, N$ do

$$
\text { 3.1. } \begin{aligned}
& \alpha_{k}=p_{k}^{\mathrm{T}} r / p_{k}^{\mathrm{T}} q_{k}, \\
& x=x+\alpha_{k} p_{k}, \\
& r=r-\alpha_{k} q_{k} ;
\end{aligned}
$$

3.2. $p_{k+1}=r$,

$q_{k+1}=A p_{k+1}$,

For $i=1, \ldots, k$ do

$$
\begin{aligned}
& \beta_{i}=-p_{i}^{\mathrm{T}} q_{k+1} / p_{i}^{\mathrm{T}} q_{i}, \\
& p_{k+1}=p_{k+1}+\beta_{i} p_{i}, \\
& q_{k+1}=q_{k+1}+\beta_{i} q_{i} .
\end{aligned}
$$

Catabriga et al. [3] introduced an algorithm similar to Algorithm 4.1, but with restart as in the GMRES algorithm implemented by Shakib et al. [11]. In this paper we consider the same restart scheme applied to Algorithm 4.2. The new algorithm is given below.

\section{Algorithm $4.3(\operatorname{LCD}(k))$}

1. Given $x, A, b, l_{\max }, k_{\max }$ and $\eta$

2. $r=b-A x$

3. $\varepsilon=\eta\|r\|$

4. Choose $p_{1}$ such that $p_{1}^{\mathrm{T}} A p_{1} \neq 0$

5. For $l=1, \ldots, l_{\max }$ do

5.1. $q_{1}=A p_{1}$

5.2. For $k=1, \ldots, k_{\max }$ do

$$
\text { 5.2.1. } \begin{aligned}
\alpha_{k}=p_{k}^{\mathrm{T}} r / p_{k}^{\mathrm{T}} q_{k} \\
\\
x=x+\alpha_{k} p_{k} \\
r=r-\alpha_{k} q_{k}
\end{aligned}
$$

5.2.2. if $\|r\|<\varepsilon$ then exit loop $k$ and $l, x$ is the solution.

5.2.3. $p_{k+1}=r$

$$
\begin{aligned}
& q_{k+1}=A p_{k+1} \\
& \text { For } i=1, \ldots, k \text { do } \\
& \beta_{i}=-p_{i}^{\mathrm{T}} q_{k+1} / p_{i}^{\mathrm{T}} q_{i} \\
& p_{k+1}=p_{k+1}+\beta_{i} p_{i} \\
& q_{k+1}=q_{k+1}+\beta_{i} q_{i}
\end{aligned}
$$

5.3. Choose the new $p_{1}$ such that $p_{1}^{\mathrm{T}} A p_{1} \neq 0$, 
where $l_{\max }$ is the maximum number of iterations, $k_{\max }$ is the number of left conjugate directions considered for each restart. Note that the number of left conjugate direction vectors was considered equal to the number of restarts. In principle both can be different. We need to store $2 k_{\max } N$-dimensional vectors $\left\{p_{1}, \ldots, p_{k_{\max }}\right\}$ and $\left\{q_{1}, \ldots, q_{k_{\max }}\right\}$. For each iteration $l$ we need only one matrix-vector product as in the GMRES algorithm. To start $\operatorname{LCD}\left(k_{\max }\right)$, we have to choose $p_{1}$ and the subsequent $p_{1}$ for each $k_{\max }$ iteration. Catabriga et al. [3] reported numerical experiments about this choice. The best results were $p_{1}=r$ for $l=1$ and $p_{1}=p_{k_{\max }+1}$ for $l=2,3, \ldots$, and in this work we adopt this choice.

\section{NUMERICAL RESULTS}

In this section, we evaluate the LCD algorithm implemented by Yuan et al. [2] and Dai and Yuan [6]. All results were implemented using restarts unless stated otherwise. The LCD algorithm given by Yuan et al. [2] is denoted by $\mathrm{LCD}_{\mathrm{A}}$ and the algorithm in Reference [6] is denoted by $\mathrm{LCD}_{\mathrm{B}}$.

\subsection{Pure convection problem}

We consider a pure convection of a scalar on a square domain, where convection is skew to the mesh and the diffusivity is negligible. Figure 1 shows the problem set up. The domain is the unit square $\Omega=[0,1] \times[0,1]$ and the boundary conditions are

$$
\begin{aligned}
& u=0.0 \text { along } y=0.0 \\
& u=0.0 \text { along } x=0.0 \text { and } 0.0<y<0.25 \\
& u=1.0 \text { along } x=0.0 \text { and } 0.25<y<1.0
\end{aligned}
$$

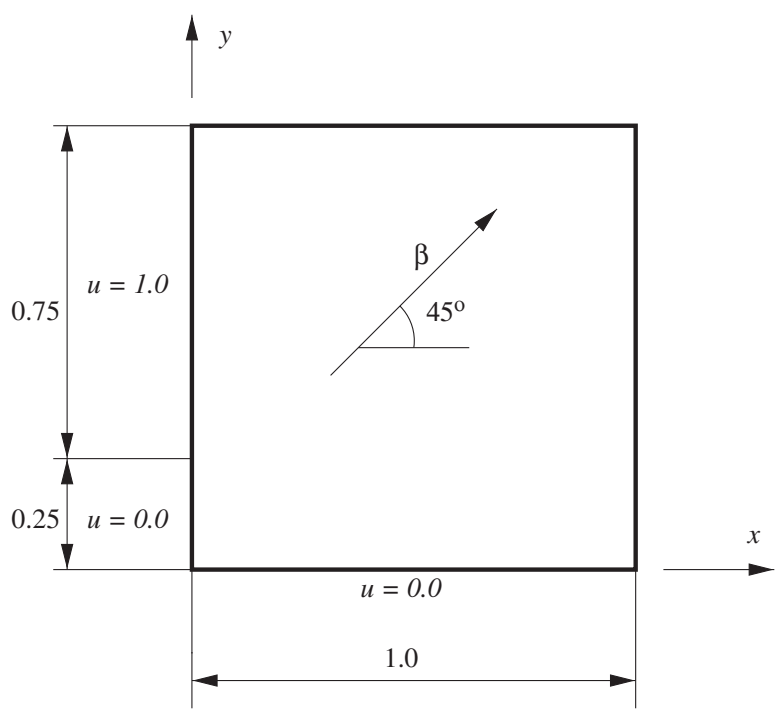

Figure 1. Problem set up - pure convection of a scalar on a square domain. 
The diffusivity is $\kappa_{x}=\kappa_{y}=1 \times 10^{-7}$, the flow direction is $45^{\circ}$ from the $x$-axis, $\|\beta\|=1$ and the stabilization parameter is computed as in Reference [7]. The domain is discretized by grids of triangular elements with $64 \times 64,128 \times 128,256 \times 256$ and $512 \times 512$ cells. Each cell is subdivided into four triangles. For these meshes we studied the influence of restart, considering several test cases, from no restart until a restart every 40 iterations. We noted that the solutions computed by $\mathrm{LCD}_{\mathrm{A}}, \mathrm{LCD}_{\mathrm{B}}$ and GMRES are virtually identical for any numbers of left conjugate direction vectors.

Table I shows the number of iterations (Niter) and CPU times for the GMRES and LCD methods using a relative residual tolerance of $10^{-10}$. In this table $N$ eq is the number of the unknowns. We can observe in Table I that the $\mathrm{LCD}_{\mathrm{A}}$ and $\mathrm{LCD}_{\mathrm{B}}$ methods converge with less iterations than GMRES in all cases, except in the case without restart. The $\mathrm{LCD}_{\mathrm{B}}$ algorithm is faster than the $\mathrm{LCD}_{\mathrm{A}}$ in most cases. Only when we consider 10 or less vectors to restart the $\mathrm{LCD}_{\mathrm{B}}$ algorithm is faster than the GMRES algorithm. Figure 2 compares the relative residual evolution for $\operatorname{LCD}(5)_{\mathrm{A}}, \operatorname{LCD}(5)_{\mathrm{B}}$ and $\operatorname{GMRES}(5)$ for the four meshes defined before. Although the relative residual in $\operatorname{LCD}(5)_{\mathrm{A}}$ and $\operatorname{LCD}(5)_{\mathrm{B}}$ decrease more slowly than in GMRES(5) in the beginning of the process, the total number of $\operatorname{LCD}(5)_{\mathrm{A}}$ and $\operatorname{LCD}(5)_{\mathrm{B}}$ iterations are almost the same and it is smaller than the number of GMRES(5) iterations in all cases. Results for the other number of restart vectors are similar.

\subsection{Non-linear convection-diffusion problem}

We consider Equation (5) with homogeneous Dirichlet boundary conditions on the unit square $(0,1) \times(0,1)$. Function $f$ has been constructed so that the exact solution was the discretization of $u(x, y)=10 x y(1-x)(1-y) e^{x^{4.5}}$. We set $\phi=20, \mathbf{u}^{0}=0, \tau_{\mathrm{a}}=10^{-9}$ and $\tau_{\mathrm{r}}=10^{-12}$. In this problem, we use the global Gauss-Seidel preconditioner for all cases and three types of forcing term (fixed, Papadrakakis and Kelley). We observed that $\mathrm{LCD}_{\mathrm{A}}, \mathrm{LCD}_{\mathrm{B}}$ and $\mathrm{GMRES}$ solutions are virtually identical for any numbers of left conjugate gradient vectors. For the following discussions, we consider the domain discretized on $512 \times 512$ cells.

Table II shows the number of linear iterations (Niter) and CPU times for the $\mathrm{LCD}_{\mathrm{A}}, \mathrm{LCD}_{\mathrm{B}}$ and GMRES methods using three types of forcing term (fixed, Papadrakakis and Kelley). We can observe that the inexact methods decrease the number of linear iterations when compared with the fixed tolerance criterion. The Papadrakakis criterion needs less inner linear iterations for all cases, except for GMRES(5). Now the $\mathrm{LCD}_{\mathrm{A}}$ algorithm is faster than $\mathrm{LCD}_{\mathrm{B}}$ for almost all number of restart vectors tested. This occurs because the calculation of two matrix-vector products per iteration is not the dominant cost in these finite difference experiments. Moreover, LCD tends to be slower than GMRES as the number of basis vectors increase. The best results in terms of CPU time using 20 restart vectors was for GMRES method with all types of forcing terms. When 40 restart vectors are considered, the best results are for GMRES, with the exception of the fixed tolerance case, where $\mathrm{LCD}_{\mathrm{A}}$ is better than GMRES.

Figures 3(a)-(c) show plots of the non-linear residual norm and Figures 4(a)-(c) show the number of linear iterations in each non-linear iteration obtained using $\operatorname{LCD}(10)_{\mathrm{A}}, \operatorname{LCD}(10)_{\mathrm{B}}$ and GMRES(10). We can observe that the fixed forcing term needs less non-linear iterations for convergence than the other two criteria, but it needs more linear iterations for each non-linear iteration. Table II shows that, for all numbers of restart vectors tested, larger 
Table I. Computational costs_-pure convection of a scalar on a square domain.

\begin{tabular}{|c|c|c|c|c|c|c|c|}
\hline \multicolumn{8}{|c|}{1 Vector } \\
\hline \multicolumn{2}{|c|}{ Mesh } & \multicolumn{2}{|c|}{ GMRES(1) } & \multicolumn{2}{|c|}{$\operatorname{LCD}(1)_{\mathrm{A}}$} & \multicolumn{2}{|c|}{$\operatorname{LCD}(1)_{\mathrm{B}}$} \\
\hline Cells & $N e q$ & Niter & Time (s) & Niter & Time (s) & Niter & Time (s) \\
\hline $64 \times 64$ & 8192 & 714 & 7 & 654 & 7 & 654 & 7 \\
\hline $128 \times 128$ & 32768 & 1123 & 55 & 1042 & 53 & 1041 & 54 \\
\hline $256 \times 256$ & 131072 & 1918 & 385 & 1784 & 364 & 1784 & 371 \\
\hline
\end{tabular}

\begin{tabular}{|c|c|c|c|c|c|c|c|}
\hline \multicolumn{2}{|c|}{ Mesh } & \multicolumn{2}{|c|}{ GMRES(5) } & \multicolumn{2}{|c|}{$\operatorname{LCD}(5)_{\mathrm{A}}$} & \multicolumn{2}{|c|}{$\operatorname{LCD}(5)_{\mathrm{B}}$} \\
\hline Cells & $N e q$ & Niter & Time (s) & Niter & Time (s) & Niter & Time ( $\mathrm{s}$ \\
\hline $64 \times 64$ & 8192 & 471 & 3 & 328 & 3 & 328 & 2 \\
\hline $128 \times 128$ & 32768 & 888 & 31 & 618 & 33 & 620 & 25 \\
\hline $256 \times 256$ & 131072 & 1661 & 238 & 1163 & 244 & 1182 & 186 \\
\hline $512 \times 512$ & 524288 & 3104 & 1726 & 2384 & 2086 & 2378 & 1533 \\
\hline
\end{tabular}

10 Vectors

\begin{tabular}{|c|c|c|c|c|c|c|c|}
\hline \multicolumn{2}{|c|}{ Mesh } & \multicolumn{2}{|c|}{ GMRES(10) } & \multicolumn{2}{|c|}{$\operatorname{LCD}(10)_{\mathrm{A}}$} & \multicolumn{2}{|c|}{$\operatorname{LCD}(10)_{\mathrm{B}}$} \\
\hline Cells & $N$ eq & Niter & Time (s) & Niter & Time (s) & Niter & Time (s) \\
\hline $64 \times 64$ & 8192 & 399 & 2 & 356 & 4 & 356 & 2 \\
\hline $128 \times 128$ & 32768 & 751 & 28 & 608 & 35 & 611 & 28 \\
\hline $256 \times 256$ & 131072 & 1479 & 220 & 1091 & 258 & 1105 & 204 \\
\hline
\end{tabular}

20 Vectors

\begin{tabular}{|c|c|c|c|c|c|c|c|}
\hline \multicolumn{2}{|c|}{ Mesh } & \multicolumn{2}{|c|}{ GMRES(20) } & \multicolumn{2}{|c|}{$\operatorname{LCD}(20)_{\mathrm{A}}$} & \multicolumn{2}{|c|}{$\operatorname{LCD}(20)_{\mathrm{B}}$} \\
\hline Cells & $N$ eq & Niter & Time (s) & Niter & Time (s) & Niter & Time (s) \\
\hline $64 \times 64$ & 8192 & 448 & 3 & 401 & 5 & 401 & 3 \\
\hline $128 \times 128$ & 32768 & 756 & 34 & 655 & 44 & 655 & 39 \\
\hline $256 \times 256$ & 131072 & 1383 & 255 & 1123 & 307 & 1150 & 285 \\
\hline
\end{tabular}

40 Vectors

\begin{tabular}{|c|c|c|c|c|c|c|c|}
\hline \multicolumn{2}{|c|}{ Mesh } & \multicolumn{2}{|c|}{ GMRES(40) } & \multicolumn{2}{|c|}{$\operatorname{LCD}(40)_{\mathrm{A}}$} & \multicolumn{2}{|c|}{$\operatorname{LCD}(40)_{\mathrm{B}}$} \\
\hline Cells & $N$ eq & Niter & Time (s) & Niter & Time (s) & Niter & Time (s) \\
\hline $64 \times 64$ & 8192 & 595 & 5 & 478 & 7 & 478 & 6 \\
\hline $128 \times 128$ & 32768 & 942 & 60 & 829 & 71 & 829 & 75 \\
\hline $256 \times 256$ & 131072 & 1552 & 405 & 1265 & 439 & 1269 & 467 \\
\hline \multicolumn{8}{|c|}{ Without restart } \\
\hline \multicolumn{2}{|c|}{ Mesh } & \multicolumn{2}{|c|}{ GMRES } & \multicolumn{2}{|c|}{$\mathrm{LCD}_{\mathrm{A}}$} & \multicolumn{2}{|c|}{$\mathrm{LCD}_{\mathrm{B}}$} \\
\hline Cells & $N$ eq & Niter & Time (s) & Niter & Time (s) & Niter & Time (s) \\
\hline $64 \times 64$ & 8192 & 261 & 6 & 262 & 11 & 262 & 13 \\
\hline $128 \times 128$ & 32768 & 533 & 320 & 534 & 307 & 538 & 481 \\
\hline
\end{tabular}


(a)
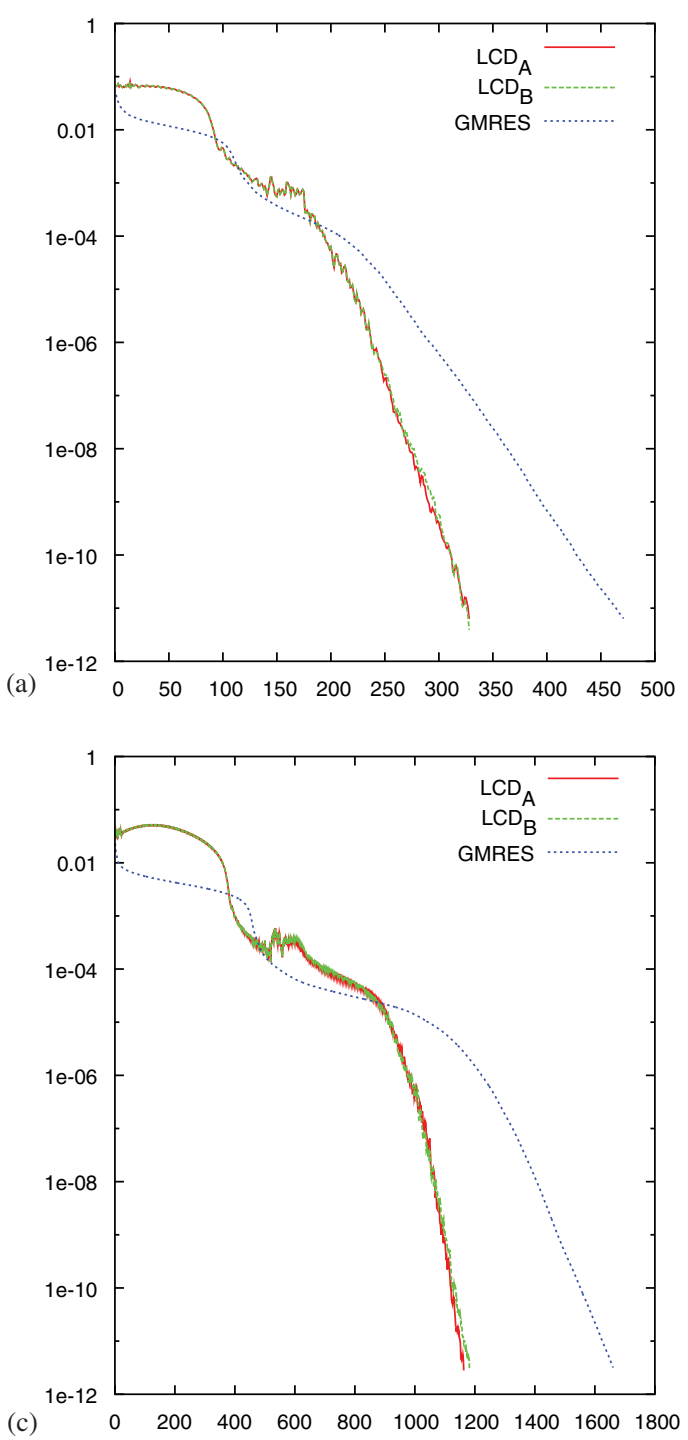

(b)

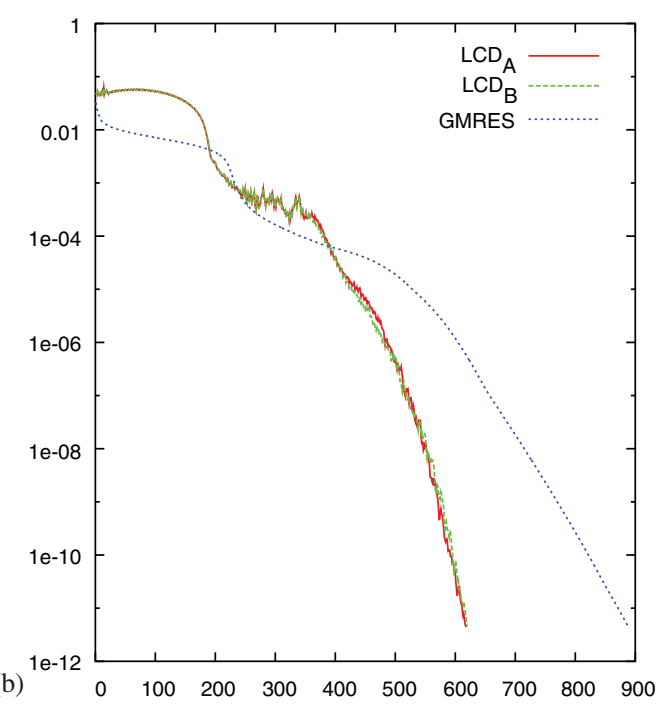

(d)

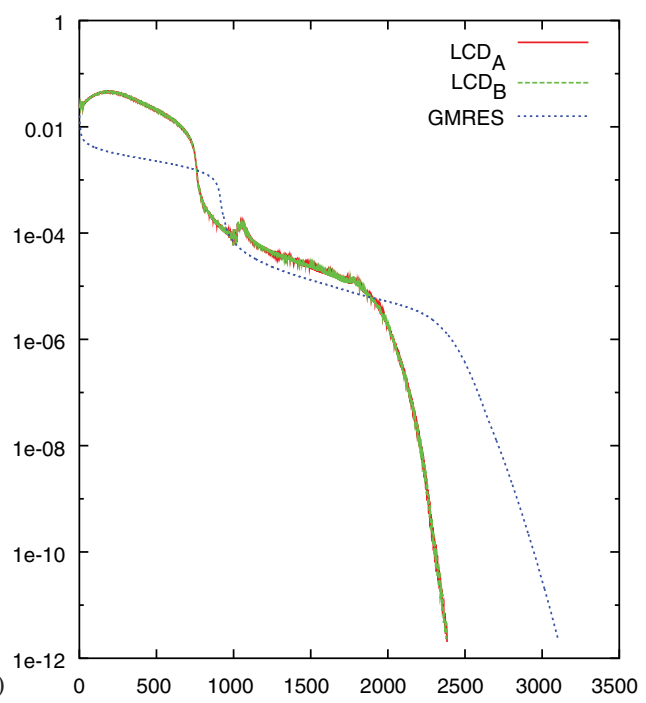

Figure 2. Relative residual evolution for $\operatorname{LCD}(5)_{\mathrm{A}}, \operatorname{LCD}(5)_{\mathrm{B}}$ and $\operatorname{GMRES}(5)$ - pure convection of a scalar on a square domain: (a) mesh $64 \times 64$; (b) mesh $128 \times 128$;

(c) mesh $256 \times 256$; and (d) mesh $512 \times 512$.

numbers of linear iterations are observed when the forcing term is fixed. Further, we can see in Figure 4 that when the Papadrakakis criterion is selected, less linear iterations are needed, which implies in less processing time. This may be due to the adaptative choice of tolerance in the inexact Newton method. 
Table II. Computational costs-mesh with $512 \times 512$ cells-non-linear convection-diffusion problem.

\begin{tabular}{|c|c|c|c|c|c|c|}
\hline \multicolumn{7}{|c|}{5 Vectors } \\
\hline \multirow[b]{2}{*}{$\eta_{k}$} & \multicolumn{2}{|c|}{ GMRES(5) } & \multicolumn{2}{|c|}{$\operatorname{LCD}(5)_{\mathrm{A}}$} & \multicolumn{2}{|c|}{$\operatorname{LCD}(5)_{\mathrm{B}}$} \\
\hline & Niter & Time (min) & Niter & Time $(\min )$ & Niter & Time $(\min )$ \\
\hline Fixed $\left(10^{-5}\right)$ & 35834 & 103.18 & 8835 & 38.73 & 8833 & 38.36 \\
\hline Papadrakakis & 7063 & 21.41 & 4214 & 18.35 & 4214 & 18.55 \\
\hline Kelley & 6214 & 18.63 & 12614 & 54.23 & 12614 & 54.73 \\
\hline \multicolumn{7}{|c|}{10 Vectors } \\
\hline & \multicolumn{2}{|c|}{ GMRES(10) } & \multicolumn{2}{|c|}{$\operatorname{LCD}(10)_{\mathrm{A}}$} & \multicolumn{2}{|c|}{$\operatorname{LCD}(10)_{\mathrm{B}}$} \\
\hline$\eta_{k}$ & Niter & Time (min) & Niter & Time (min) & Niter & Time $(\min )$ \\
\hline Fixed $\left(10^{-5}\right)$ & 17903 & 66.00 & 4907 & 25.36 & 4907 & 28.75 \\
\hline Papadrakakis & 3144 & 11.41 & 1754 & 9.08 & 1754 & 10.53 \\
\hline Kelley & 3444 & 12.76 & 3243 & 16.83 & 3243 & 17.93 \\
\hline \multicolumn{7}{|c|}{20 Vectors } \\
\hline & \multicolumn{2}{|c|}{ GMRES(20) } & \multicolumn{2}{|c|}{$\operatorname{LCD}(20)_{\mathrm{A}}$} & \multicolumn{2}{|c|}{$\operatorname{LCD}(20)_{\mathrm{B}}$} \\
\hline$\eta_{k}$ & Niter & Time (min) & Niter & Time $(\min )$ & Niter & Time $(\min )$ \\
\hline Fixed $\left(10^{-5}\right)$ & 9098 & 47.15 & 12809 & 87.71 & 12809 & 116.33 \\
\hline Papadrakakis & 1721 & 9.10 & 1414 & 9.93 & 1414 & 12.11 \\
\hline Kelley & 2450 & 13.10 & 2132 & 14.68 & 2132 & 19.36 \\
\hline \multicolumn{7}{|c|}{40 Vectors } \\
\hline & \multicolumn{2}{|c|}{ GMRES(40) } & \multicolumn{2}{|c|}{$\mathrm{LCD}(40)_{\mathrm{A}}$} & \multicolumn{2}{|c|}{$\operatorname{LCD}(40)_{\mathrm{B}}$} \\
\hline$\eta_{k}$ & Niter & Time (min) & Niter & Time $(\min )$ & Niter & Time $(\min )$ \\
\hline Fixed $\left(10^{-5}\right)$ & 4495 & 39.45 & 2962 & 29.96 & 2962 & 43.20 \\
\hline Papadrakakis & 1319 & 10.45 & 1481 & 14.03 & 1481 & 22.06 \\
\hline Kelley & 1650 & 18.83 & 1678 & 22.10 & 1678 & 25.70 \\
\hline
\end{tabular}

\section{CONCLUSION}

In this work, we compared the performance of LCD and GMRES algorithms in the finite element and finite difference solutions for linear and non-linear convection-diffusion problems. The non-linear problem solution have been carried out by the inexact Newton method. We studied two choices for the forcing term of the inexact Newton method. We implemented two different algorithms for the LCD method. One considers two matrix-vector products per iteration $\left(\mathrm{LCD}_{\mathrm{A}}\right)$ and the other considers only one matrix-vector product, but needs to compute more inner products, if we consider $k$ vectors to restart $\left(\mathrm{LCD}_{\mathrm{B}}\right)$. 

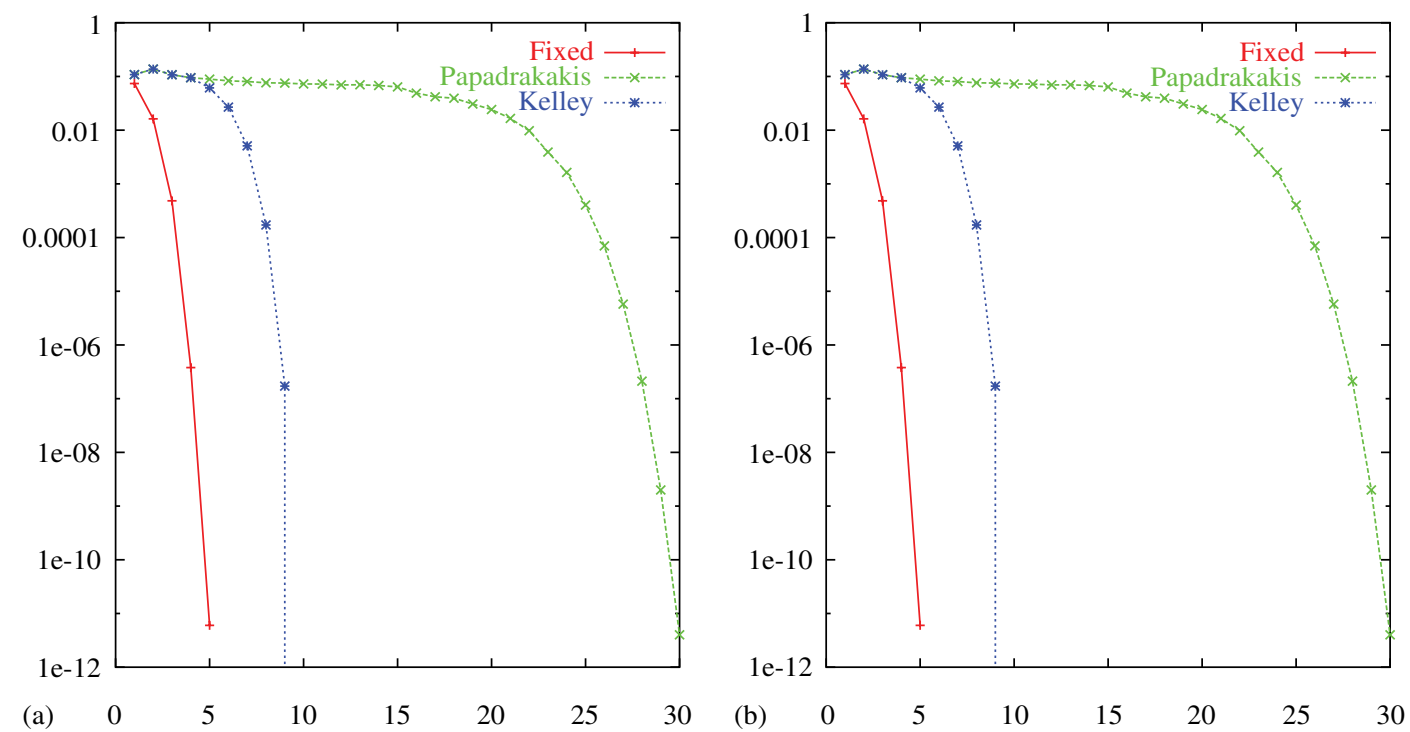

(a) $\begin{array}{lllllll}0 & 5 & 10 & 15 & 20 & 25 & 30\end{array}$

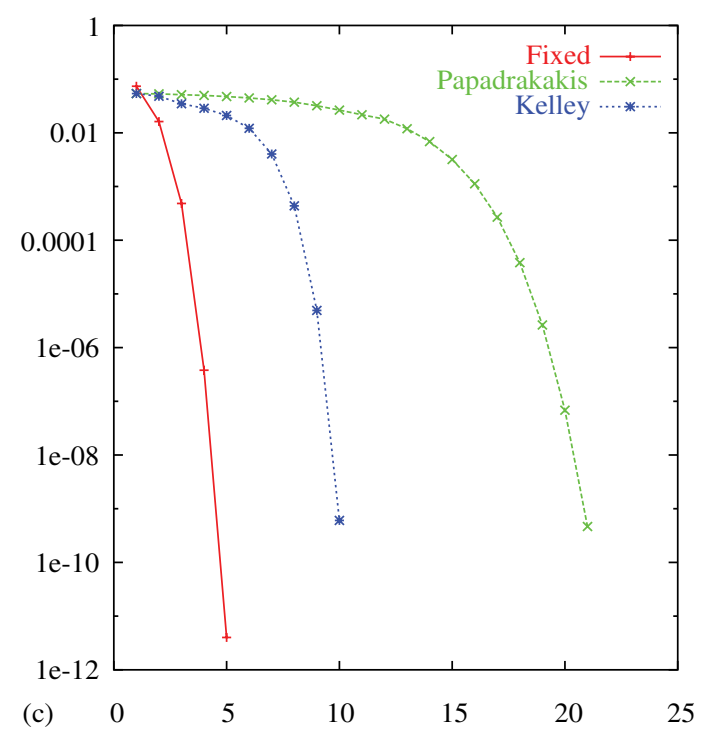

Figure 3. Convergence histories using different choices of the forcing terms (Non-linear iterations $\left.\times\left\|F\left(u^{k}\right)\right\|\right)$-mesh with $512 \times 512$ cells-non-linear convection-diffusion problem:

(a) $\operatorname{LCD}(10)_{\mathrm{A}}$ solution; (b) $\operatorname{LCD}(10)_{\mathrm{B}}$ solution; and (c) GMRES (10) solution.

For the finite element experiments we can observe that the $\mathrm{LCD}_{\mathrm{B}}$ algorithm is faster than GMRES and $\mathrm{LCD}_{\mathrm{A}}$ only when we consider small number of basis vectors. For the finite difference experiments, the $\mathrm{LCD}_{\mathrm{A}}$ is faster than $\mathrm{LCD}_{\mathrm{B}}$ for all cases. $\mathrm{LCD}_{\mathrm{A}}$ using 10 vectors to restart and Papadrakakis criterion gave the smallest CPU time. In general, GMRES is faster 

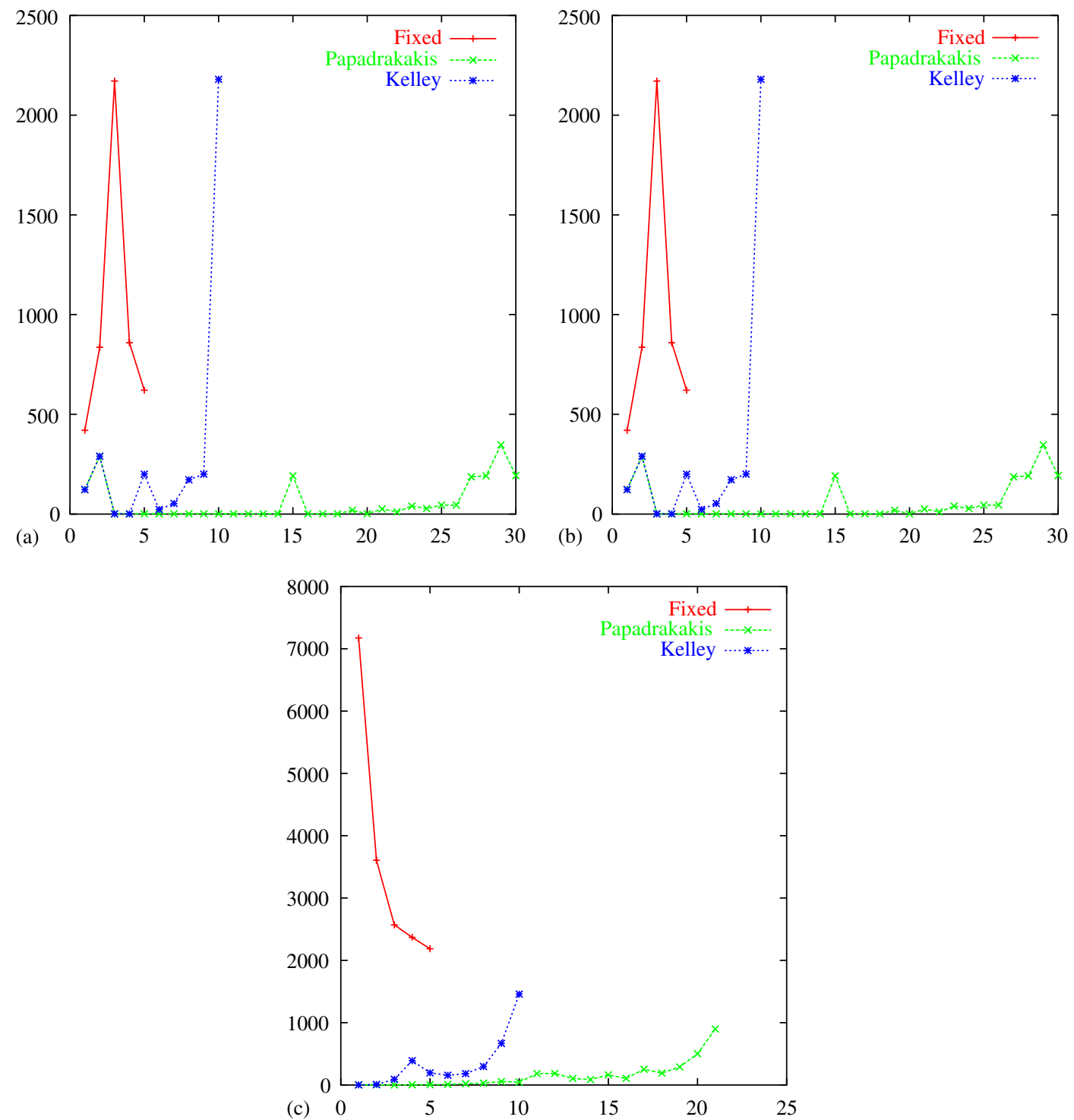

Figure 4. Number of linear iteration behaviour using different choices of the forcing terms - mesh with $512 \times 512$ cells - non-linear convection-diffusion problem: (a) $\operatorname{LCD}(10)_{\mathrm{A}}$ solution; (b) $\operatorname{LCD}(10)_{\mathrm{B}}$ solution; and (c) GMRES (10) solution.

than LCD when the number of restart vectors are increased. On the criteria to choose the forcing term for the inexact Newton method, we conclude that Papadrakakis criterion was the best option in all of our experiments. 
The present numerical experiments are not exhaustive and certainly more tests are required. The apparent superiority of $\mathrm{LCD}_{\mathrm{A}}$ in the finite difference test case should be further explored, and these will addressed in a forthcoming paper.

\section{ACKNOWLEDGEMENTS}

We would like to thank the support of $\mathrm{CNPq} / \mathrm{MCT}$ and the Federal University of Espírito Santo under the PIBIC/PIVIC program. Partial support of the CAPES-University of Texas at Austin International Cooperation Program is gratefully acknowledged.

\section{REFERENCES}

1. Saad Y. Iterative Methods for Sparse Linear Systems. PWS Publishing: Boston, MA, 1996.

2. Yuan JY, Golub GH, Plemmons RJ, Cecílio WAG. Semiconjugate direction methods for real positive definite systems. BIT Numerical Mathematics 2004; 44(1):189-207.

3. Catabriga L, Coutinho ALGA, Franca LP. Evaluating the LCD algorithm for solving linear systems of equations arising from implicit SUPG formulation of compressible flows. International Journal for Numerical Methods in Engineering 2004; 60:1513-1534.

4. Catabriga L, Coutinho ALGA. Implicit SUPG solution of Euler equations using edge-based data structures. Computer Methods in Applied Mechanics and Engineering 2002; 191:3477-3490.

5. Valentim EC, Pessoa LM, Melotti BZ, Valli AMP, Catabriga L. Comparison between GMRES and LCD methods in the implementation of an inexact Newton method (in Portuguese). 10th Brazilian Congress of Thermal Engineering and Sciences CD-ROM, Rio de Janeiro, Brazil, 2004; 1-12.

6. Dai Y, Yuan JY. Study on semi-conjugate direction methods for non-symmetric systems. International Journal for Numerical Methods in Engineering 2004; 60:1383-1399.

7. Brooks AN, Hughes TJR. Streamline Upwind/Petrov-Galerkin formulations for convection dominated flows with particular emphasis on the incompressible Navier-Stokes equations. Computer Methods in Applied Mechanics and Engineering 1982; 32:199-259.

8. Kelley CT. Iterative Methods for Linear and Non-linear Equations. SIAM: Philadelphia, PA, 1995.

9. Pernice M, Walker HF. NITSOL: a Newton iterative solver for non-linear system. SIAM Journal on Scientific Computing 1998; 19:302-318.

10. Papadrakakis M. Solving Large-Scale Problems in Mechanics: The Development and Application of Computational Solution Procedures. Wiley: London, 1993.

11. Shakib F, Hughes TJR, Johan Z. A multi-element group preconditioned GMRES algorithm for nonsymmetric systems arising in finite element analysis. Computer Methods in Applied Mechanics and Engineering 1989; 65:415-456. 